\title{
Avances en Neurobiología de la Conducta
}

\section{Advances in Neurobiology of Behavior}

\author{
Luis Alberto Raggi S. ${ }^{1}$ \\ Universidad de Chile-Universidad Santo Tomás de Chile \\ Ingrid Rojas $\mathrm{S}^{2}$ \\ Universidad Santo Tomás de Chile
}

(Recepción: Junio 2006 - Aceptación: Septiembre 2006)

\begin{abstract}
La experiencia diaria nos enseña que el cerebro tiene una notable capacidad de adaptarse a cambios ambientales, de almacenar memoria y determinar la conducta. Pero hasta que punto esta adaptación del cerebro adulto, depende de reordenamientos en las conexiones entre las células nerviosas, sigue siendo uno de los mayores desafios de la neurobiología moderna. Los mecanismos sinápticos de la plasticidad en la corteza adulta, dependiente de la experiencia, aún se desconocen. En esta breve revisión se comunican algunos de los avances en la ncurobiología de la plasticidad neuronal.
\end{abstract}

Palabras clave: Neurobiología, sinapsis, plasticidad ncuronal.

\begin{abstract}
Everyday experience tells us that brain have a remarkable capacity to adapt to changes in the environment and to form memories and determine behaviour. But to what extent does this malleability of the adult brain depend on physical rearrangements of the connections between nerve cells is one of the most challenging questions in modern neurobiology. The cellular and synaptic mechanisms underlying experience-dependent plasticity in the adult neocortex is not understood. Here we report some advances in neurobiology of neuron plasticity.
\end{abstract}

Key words: Neurobiology, synapses, neuron plasticity.

\section{Introducción}

El cerebro humano es una red de más de 100.000 millones de neuronas individuales interconectadas en sistemas que elaboran y constituyen el sustrato de nuestra percepción del mundo exterior, la acción, las emociones y el aprendizaje; he aquí el grado de importancia que tiene la comprensión de los determinantes de la conducta, tanto innatos (genéticos) como ambientales, pues obviamente lo que se hereda no es la conducta, sino que el ADN, responsable de codificar proteínas importantes para el desarrollo y la regulación de los circuitos nerviosos que subyacen a la conducta; el ambiente, que comienza a ejercer su influencia en el útero materno, toma una gran importancia después del nacimiento.

Es aquí donde adquiere una relevancia significativa la neurociencia moderna, pues al re-

\footnotetext{
' Luis Alberto Raggi Saini, Casilla 2, Correo 15, Santiago. Iraggi@uchilc.cl. Universidad de Chile. Fono (56-2) 9785530, Fax (56-2) 9785526. Profesor Titular, Doctor en Veterinaria, Universidad de Córdoba, España.

= Ingrid Rojas Stuardo, psicoingeba@hotmail.com. Universidad Santo Tomás de Chilc. Alumna quinto año de Psicologia. Ayudante Psicobiología.
}

presentar una fusión de la biología molecular, neuropsicofisiología, anatomía, embriología, biología celular y psicología, refuerza la idea que el estudio adecuado de la mente, empieza por el estudio del cerebro. La psicología cognitiva y la psicoterapia clínica, pueden fortalecerse en la actualidad con los conocimientos sobre neurobiología celular de la conducta. La tarea de los años venideros es crear una psicología que, aunque siga ocupándose de los problemas relativos a cómo se generan las representaciones internas, la psicodinámica y los estados subjetivos de la mente, tenga un sólido fundamento en la neurociencia (Kandel et al. 2001).

\section{Bases neurobiológicas}

En la actualidad, el conocimiento del cerebro y su funcionamiento se estudia con tecnologías muy avanzadas, como la imagenología óptica de señales intrínsecas (IOS), que monitorean el aumento en el flujo sanguíneo, que es un indicador de la actividad cognitiva de los circuitos neuronales que participan, por ejemplo, en la generación de una respuesta a una determinada pregunta y pone en evidencia el proceso llevado a cabo en cada área específica, en el caso 
de la respuesta verbal a una pregunta, el área de Broca. De la misma forma, la resonancia magnética funcional registra los aumentos del flujo sanguíneo como una medida indirecta de la actividad cognitiva, pero a diferencia de la IOS es no invasiva y por lo tanto, puede usarse para estudiar la función cerebral en una gran cantidad de individuos y explorar así, los circuitos neuronales de pacientes con depresión, dislexia, esquizofrenia y otros trastornos neurológicos, además de aplicarse en individuos sanos que realizan una determinada tarea o función.

Tomas Willis, contemporáneo de Descartes, fue el primero en sugerir que el cerebro era el asiento de la mente y que diferentes partes daban origen a funciones cognitivas específicas. De esta forma, como concepto, la frenología del siglo XIX se anticipó de manera notable, aunque erradamente, a los tiempos actuales.

En la última década se ha confirmado que diferentes funciones tienen lugar en ubicaciones específicas, aun cuando las funciones cognitivas no pueden ubicarse en puntos precisos, sino más bien en una compleja red de circuitos que interactúan entre sí, produciendo un cerebro único para cada individuo.

¿Cómo se organizan, maduran y funcionan los circuitos neuronales? Entre los descubrimientos más notables de los últimos tiempos, se encuentra la identificación de compuestos propios del medioambiente embrionario, capaces de atraer o repeler las terminaciones axónicas. Los factores con actividad de atracción axonal, son las cadherinas y las moléculas de adhesión celular o CAM, y las moléculas de atracción por difusión denominadas netrinas. Las moléculas o factores con capacidad para repeler los axones, actúan bajo el principio de repulsión por difusión, como las semaforinas y tenasinas y de repulsión por contacto como los ligandos Eph y las semaforinas de dominio transmembranal (Ghosh, 1997; Holmberg et al. 2000).

Los conocimientos actuales permiten concluir que el crecimiento de las terminaciones nerviosas está guiado por fuerzas de atracción y repulsión, a las que cada uno de los millones de axones responde de manera diferente. Sistema que, aunque complejo, tiene un mínimo margen de error si no es intervenido por factores externos.

La organización en tractos y fascículos, ejerce una serie de fuerzas de tensión mecánica, que traccionan a la corteza y conduce al desarrollo de surcos y circunvoluciones.

Si bien es cierto que la experiencia sensorial es un requisito fundamental para la formación de sinapsis efectivas entre neuronas, los estímulos sensoriales por sí mismos no son suficientes para establecer un circuito activo. La corteza alcanza un alto grado de maduración al nacimiento y por ello es factible pensar que la estructura básica del cerebro se forma durante la etapa prenatal, pero gracias a un alto grado de plasticidad, durante la vida, las redes neuronales se modifican permanentemente.

También hay coincidencia entre los investigadores de que existen periodos críticos del desarrollo, durante los cuales los estímulos sensoriales externos son vitales para que la maduración se ejecute con éxito y que, para la construcción de patrones sinápticos adultos, es necesario eliminar una enorme cantidad de conexiones inmaduras y adicionar circuitos neuronales nuevos, mediados por señales intracelulares que modifican la arquitectura de la neurona y promueve el desarrollo de nuevas sinapsis.

La plasticidad neuronal y por ello del cerebro, queda manifiesta cuando se observa su capacidad para volver a moldearse y reorganizarse durante la edad adulta.

Nuestro cerebro tiene una notoria capacidad de adaptarse a los cambios del ambiente y de almacenar memoria. De acuerdo a lo señalado por Ottersen y Helm (2002), uno de los desafíos mayores de la neurobiología, es responder a la pregunta de si esta plasticidad del cerebro adulto, depende de reordenamientos de las conexiones entre las células nerviosas.

De acuerdo a lo señalado por Trachtenberg et al. (2002), los cambios neuronales producto de la experiencia en el adulto, no dependen de modificaciones en la ramificación de dendritas o cambios en los axones, los que se mantienen estables después de varios días de estimulación; sin embargo, la aparición y desaparición de espinas dendríticas está muy ligada a la estimulación y la experiencia, por ello es que proponen que los cambios adaptativos en la neocorteza del adulto dependen de la aparición y desaparición de las espinas dendríticas, sin que ocurran modificaciones en los otros procesos neuronales.

De acuerdo a estos autores, los periodos críticos son ventanas temporales en el desarrollo 
post natal, donde la experiencia influye profundamente en la organización final de los contactos neuronales, generándose una acelerada sinaptogénesis y también una eliminación de sinapsis, siendo todos estos cambios locales. Esto asegura que la plasticidad de la corteza cerebral del adulto sea reversible, debido a que los patrones presinápticos originales se mantienen. Sin embargo, periodos críticos "mantenidos por largo tiempo" serían irreversibles al producir cambios en las ramificaciones de las dendritas y los axones los cuales producirían una reestructuración de los circuitos.

En contraposición con lo anterior, Grutzendler et al. (2002, citados por Ottersen y Helm, 2002), postulan que las espinas dendríticas se generan producto de la experiencia, son estables en el tiempo y son las responsables de la memoria a largo plazo; por lo que el aprendizaje y la conducta tendrían como base cambios en las sinapsis ya existentes.

La plasticidad neuronal y por lo tanto del cerebro, queda manifiesta cuando se observa la capacidad para volver a moldearse y reorganizarse durante la edad adulta. Los ciegos que leen braille muestran un aumento notable en el tamaño de la corteza somatosensorial, especialmente aquella dedicada al dedo índice derecho; análogamente, los violinistas en la región asociada con los dedos de la mano izquierda. Esta plasticidad ha sorprendido incluso, a aquellos que pensaban en que se requeririan muchas generaciones para observar un cambio importante en el área cortical de las regiones somatosensorial y motora que maneja por ejemplo el dedo pulgar, ligado en la actualidad a muchas funciones relacionadas con los adelantos tecnológicos, por ejemplo los teléfonos celulares.

Los estudios futuros, están orientados a la comprensión de la sinapsis, que representaría el asiento de la memoria, de procesos complejos como la conducta, además de representar la base de diferentes trastornos, entre los que se encuentra la depresión bipolar y su correspondiente tratamiento con psicofármacos.

Un enfoque diferente, pero integrado, es la participación de las emociones en la génesis, desarrollo y recuperación de las enfermedades. La psiconeuroinmunología es la disciplina que se ocupa de la relación, entre los procesos cerebrales y la defensa biológica del organismo; conceptualización que tuvo sus primeras refe- rencias, en el siglo $\mathrm{XX}$, con las teorías psicoanalíticas de Sigmund Freud, las que señalaban a los procesos mentales como causa de trastornos orgánicos; posteriormente se desarrolló el concepto del estrés, demostrándose que ante situaciones de conflicto se producen diversos cambios fisiológicos, acuñándose el término de "psiconeuroinmunología", para asociar los trastornos del sistema inmune con los cambios psicológicos (Crossley y Morgado, 2003).

En esta línea, el estrés y la depresión han sido asociados con el empeoramiento de la función inmune y el aumento de la susceptibilidad del paciente hacia las enfermedades infecciosas y el cáncer, pues, en situaciones de ansiedad o de depresión crónica se elevan los niveles sanguíneos de cortisol, la que disminuye la respuesta inmune y colabora en la generación de un cáncer. Reforzando, lo anterior, el hecho de que los estados emocionales pueden modificar, incluso, el número y eficiencia de las células NK (eliminan células cancerígenas). Las evidencias existentes indican que estas células poseen en su membrana receptores de epinefrina, norepinefrina y neuropéptidos y que la exposición a estas moléculas inhibe su actividad.

Actualmente se piensa que la depresión está asociada con la activación de algunos aspectos de la inmunidad celular, que redundan en la hipersecreción de citoquinas proinflamatorias y en la hiperactividad del eje hipotálamo-hipófisisadrenal. Diversas investigaciones experimentales muestran que esta activación inmune induce cambios de comportamiento y neuroquímicos "de tipo estrés", que evidencian una integración de los sistemas inmune, endocrino y de neurotransmisores, concluyendo que el resultado de esta integración puede estar involucrado a nivel causal en la etiología de la depresión (Leonard, 2000).

El elemento común de la psiconeuroinmunología son las emociones, actuando como un "puente real y significativo entre la mente y el organismo". La neurobiología actual, apoya tal relación considerando los planteamientos de Antonio Damasio que propone la hipótesis del "marcador somático", como los dispositivos neuronales que establecen una interrelación entre los circuitos emocionales y el razonamiento (lóbulo frontal).

Crossley y Morgado (2003) plantean que los brazos ejecutores de la emoción son el 
hipotálamo, el sistema nervioso autónomo, el sistema endocrino y el sistema inmune. Hipotálamo y amígdala están conectados mediante haces nerviosos que transmiten la información bidireccionalmente, funcionando el primero como un dispositivo homeostático, conectándose además, directamente con el sistema nervioso autónomo (estimulando potenciales de acción en receptores de norepinefrina y acetilcolina), con el sistema endocrino (eje hipotálamo, hipófisis y secreción de neuropéptidos) y con el sistema inmune (respondiendo ante una alarma inmunológica), el que al poseer receptores para epinefrina, adrenalina, cortisol y numerosas hormonas, puede aumentar o disminuir su eficacia frente a la activación del sistema nervioso autónomo y el sistema endocrino. Es precisamente la función e influencia recíproca de estos tres sistemas, los que integran y regulan las reacciones fisiológicas y la reacción emocional, pues la amígdala (neurotransmisor GABA y serotonina, el que aumenta el efecto inhibitorio del primero), a través de estos sistemas puede cambiar nuestra biología. Ejemplo de ello, es que hoy se sabe que en el proceso de reparación ósea se requiere interleucina-1 que es producida por los linfocitos bajo el estímulo de la adrenalina, la que a su vez es modificada por las emociones".

El cerebro puede actuar sobre todo el organismo, a través del sistema nervioso, hormonal e inmune, de una forma integrada y holística. Afirmación que ya sostenía Aristóteles 360 años antes de Cristo: "Un cambio en el estado de la psiquis produce un cambio en la estructura del cuerpo y un cambio en la estructura del cuerpo produce un cambio en la estructura de la mente".

Otro hallazgo, que reafirma la influencia recíproca de los sistemas orgánicos y la interdependencia de éstos con el sistema nervioso, tiene relación con la diferenciación por sexo; se sabe que bajo la influencia de las hormonas fetales circulantes, posterior a la diferenciación de los genitales internos y externos, se produce un desarrollo dimórfico de ciertas áreas del cerebro. El cerebro es ambitípico y en él prevalece el desarrollo de características femeninas, a menos que exista un nivel adecuado de andrógenos en circulación. Las funciones específicas hipotalámicas e hipofisiarias que se diferenciarán como cíclicas en la mujer y no cíclicas en el hombre, quedan determinadas por esa diferenciación. La diferenciación masculi- no/femenino del cerebro, sólo se produce en el tercer trimestre de la gestación, después de la diferenciación de los órganos sexuales externos, continuando tal vez durante el primer trimestre postnatal. En los primates la comunicación y el aprendizaje sociales tempranos, tienen una importancia dominante en la determinación de la conducta sexual; por lo tanto, el control de la conducta real de apareamiento, está en gran medida determinado por las interacciones sociales más tempranas (Kemberg, 1996).

Las características sexuales secundarias que emergen en la pubertad, son inducidas por factores del sistema nervioso central y controladas por un incremento significativo de los andrógenos o estrógenos circulantes, lo mismo que las funciones femeninas específicas. Aún se desconoce de que modo exacto el sistema nervioso afecta el inicio de la pubertad; se ha considerado que uno de los mecanismos, es la reducción de la sensibilidad del hipotálamo a la retroalimentación negativa (Kemberg, 1996).

La conducta sexual es una conducta no reguladora y aparte de la influencia hormonal y el aprendizaje social temprano, se ve influenciada por el hipotálamo y la amígdala, existiendo algunas evidencias experimentales de una base neural para la orientación sexual (Kolb y Whishaw, 2002).

\section{Síntesis}

La mente es lo que el cerebro crea y cada individuo es diferente a sus semejantes. Los genes de cada individuo poseen información que inicia un programa exclusivo de desarrollo neuronal. Sin embargo, este desarrollo del sistema nervioso, incluso en el ambiente fetal, va a depender de sus interacciones con el ambiente, lo que llamamos comúnmente experiencia.

La capacidad de comportamiento de cada individuo depende de sus patrones de actividad nerviosa exclusivos, algunos de los cuales se experimentan como pensamientos, sentimientos y emociones (Pinel, 2001).

La conciencia organiza la información que penetra en el cerebro, el conocimiento que crea el cerebro y la conducta que produce el cerebro (Kolb y Whishaw, 2002).

El comportamiento presente de cada individuo "bio, psico, social", surge de la interacción entre los patrones de actividad nerviosa y su per- 


\section{AVANCES EN NEUROBIOLOGÍA DE LA CONDUCTA}

cepción de la situación actual y su éxito posibilita que sus genes pasen o no a generaciones futuras, evolución que influye sobre el conjunto de genes que afectan el comportamiento de cada especie.

\section{Referencias}

Crossley, J.; Morgado, F. (2003). De fantasmas y demonios. Editorial Grijalbo, Santiago, Chile. 182 pp.

Ghosh, A. (1997). Axons follow reeling routcs. Nature. Vol. 385, pp. 23-24.

Holmberg, J.; Clarke, D.; Frisen, J. (2000). Regulation of repulsion versus adhesion by different splice forms of an Eph receptor. Nature. Vol. 408, pp. 203-206.

Kandel, E.; Schwartz, J.; Jessell, T. (2001). Principios de Neurociencia. Cuarta Edición. McGraw-Hill Interamericana, $1400 \mathrm{pp}$.
Kemberg, O. (1996). Relaciones amorosas, normalidad y patologia. Editorial Paidós, Buenos Aircs, Barcelona, México. 336 pp.

Kolb, B.; Whishaw, I. (2002). Cerebro y conducta: una introducción. Primera Edición en Español. McGraw Hill Interamcricana, $635 \mathrm{pp}$.

Leonard, B. (2000). Depression, stress and immunological activation. World J. Biol Psychiatry. Vol. 1, pp. 17.25.

Ottersen, O. y Helm, J. (2002). How hardwired is the brain? Nature. Vol. 420, pp. 751-752.

Pinel, J. (2001). Biopsicologia. Cuarta Edición en Español. Prentice Hall, Madrid, España. 637 pp.

Trachtenberg, J.; Chen, B.; Knott, G.; Feng, G.; Sanes, J.; Welker, E.; Svoboda, K. (2002). Long-term in vivo imaging of experience-dependent synaptic plasticity in adult cortex. Nature. Vol. 420, pp.788-794. 